\title{
On the Distribution of Norm of Vector Projection and Rejection of Two Complex Normal Random Vectors
}

\author{
Mehdi Maleki and Hamid Reza Bahrami \\ Department of Electrical and Computer Engineering, The University of Akron, Akron, OH 44325, USA \\ Correspondence should be addressed to Hamid Reza Bahrami; hrb@uakron.edu
}

Received 30 July 2015; Accepted 15 October 2015

Academic Editor: Guangming Xie

Copyright (c) 2015 M. Maleki and H. R. Bahrami. This is an open access article distributed under the Creative Commons Attribution License, which permits unrestricted use, distribution, and reproduction in any medium, provided the original work is properly cited.

\begin{abstract}
Vector projection and vector rejection are highly common and useful operations in mathematics, information theory, and signal processing. In this paper, we find the distribution of the norm of projection and rejection vectors when the original vectors are standard complex normally distributed.
\end{abstract}

\section{Introduction}

Complex numbers and vectors have been used extensively in the modeling of many scientific and engineering problems. For example, in electronics, sinusoidal voltage and current signals are much better described using complex numbers sometimes called phasors [1]. Similarly, electrical impedance, the measure of the opposition that an electrical circuit presents to a current when a voltage is applied, can be modeled as a complex number that changes with the frequency of the applied voltage. Another example is in electromagnetism. Rather than trying to describe an electromagnetic field by two real quantities of electric field strength and magnetic field strength, it can be better described as a single complex number, of which the electric and magnetic components are simply the real and imaginary parts [2]. In digital communications, a modulated signal can be conveniently modeled in baseband as a complex signal that encompasses the amplitude and phase information of the original modulated signal [3].

Central limit theorem states that, under mild conditions, the arithmetic mean of many random variables independently drawn from the different distributions asymptotically converges to a normal distribution [4]. This makes the normal distribution very useful to model many continuous random events. Physical quantities that are the sum of many independent phenomena often have distributions that closely approximate a normal distribution. For example, in wireless communication, when there are many scatterer objects in the environment that scatter the radio signal before its arrival at the receiver, the received signal can be modeled as a normal random process [5].

Vector projection as well as rejection also appears in many scientific and engineering problems [6]. For example, in classical Physics, it is helpful to decompose the available forces into orthogonal and horizontal components [7]. In detection theory, it is often beneficial to project the noise vector onto the signal subspace and discard the rejection vector of noise [8]. Projection requires solving the problem of finding the vector $\widehat{\mathbf{a}}$ as the best approximation of vector $\mathbf{a}$ in the direction of vector $\mathbf{b}$. The difference of $\mathbf{a}$ and $\widehat{\mathbf{a}}$ can be considered as the rejection vector indicating the approximation error. As another example from statistical classification, consider $M$ independent complex random vectors $\mathbf{x}_{m} \in \mathbb{C}^{N}, m=$ $1, \ldots, M$, each introducing a category, and a complex random vector $\mathbf{y}$ independent of $\mathbf{x}_{m}$ 's. The norm of the projection of $\mathbf{y}$ in the direction of each of $\mathbf{x}_{m}$ vectors can be used as a metric to associate $\mathbf{y}$ with one of these categories. Also, the norm of the rejection of $\mathbf{y}$ from $\mathbf{x}_{m}$ can be considered as the error of such an approximation. In this paper, we derive the distribution of these norms assuming normal distributions for $\mathbf{x}_{m}$ 's and $\mathbf{y}$.

\section{Projection and Rejection Vectors}

Consider two complex vectors $\mathbf{a}, \mathbf{b} \in \mathbb{C}^{N}$. We can decompose any of these vectors (say a) into two parts. One part is collinear with $\mathbf{b}$ which is called vector projection of $\mathbf{a}$ on $\mathbf{b}$, 
and the other part is orthogonal to $\mathbf{b}$ which is called vector rejection of $\mathbf{a}$ from $\mathbf{b}$. Mathematically, the vector projection of $\mathbf{a}$ on $\mathbf{b}$ can be expressed as [6]

$$
\operatorname{proj}_{\mathbf{b}} \mathbf{a}=\frac{\langle\mathbf{a}, \mathbf{b}\rangle}{\langle\mathbf{b}, \mathbf{b}\rangle} \mathbf{b},
$$

where $\langle\mathbf{a}, \mathbf{b}\rangle=\mathbf{b}^{H} \mathbf{a}$. Also, the vector rejection of $\mathbf{a}$ from $\mathbf{b}$ can be written as

$$
\operatorname{rej}_{\mathbf{b}} \mathbf{a}=\mathbf{a}-\operatorname{proj}_{\mathbf{b}} \mathbf{a} .
$$

Considering these definitions, the squared norm of vector projection/rejection of a on/from $\mathbf{b}$ can be, respectively, written as

$$
\begin{aligned}
& w_{P}(\mathbf{a}, \mathbf{b})=\left\|\operatorname{proj}_{\mathbf{b}} \mathbf{a}\right\|^{2}=\frac{|\langle\mathbf{a}, \mathbf{b}\rangle|^{2}}{\|\mathbf{b}\|^{2}}, \\
& w_{R}(\mathbf{a}, \mathbf{b})=\left\|\mathrm{rej}_{\mathbf{b}} \mathbf{a}\right\|^{2}=\|\mathbf{a}\|^{2}-\frac{|\langle\mathbf{a}, \mathbf{b}\rangle|^{2}}{\|\mathbf{b}\|^{2}} .
\end{aligned}
$$

In the next section, we assume that the original vectors $\mathbf{a}, \mathbf{b} \epsilon$ $\mathbb{C}^{N}$ are complex normally distributed vectors. Our aim is to find the distribution of the norm and squared norm of both vector projection and vector rejection.

\section{Vector Projection and Rejection of Two Normal Random Vectors}

Let us assume $\mathbf{a}$ and $\mathbf{b}$ are zero mean and independent and identically distributed (i.i.d.) normal vectors such that

$$
\mathbf{c} \sim \mathbb{C} \mathcal{N}\left(\mathbf{0}_{N \times 1}, 2 \mathbf{I}_{2 N}\right),
$$

where $\mathbf{c}=\left[\mathbf{a}^{H}, \mathbf{b}^{H}\right]^{H}$. In the following, we find the distribution of the norm of vector projection and vector rejection of such random vectors.

The squared norm of vector rejection is the difference of a chi-squared random variable $\left(\|\mathbf{a}\|^{2}\right)$ and squared norm of the vector projection which is a ratio of two dependent random variables. Also, $\|\mathbf{a}\|^{2}$ and the squared norm of the vector projection are not independent. Therefore, direct calculation of the distribution of the norm of the vector rejection is difficult. Instead, in this section, we calculate the moments of $w_{R}$. To do this, we first introduce a few lemmas.

Lemma 1. For a standard normal random variable $x$, one has

$$
E\left\{|x|^{2 L}(x)^{K}\left(x^{*}\right)^{\bar{K}}\right\}= \begin{cases}(K+L) !, & K=\bar{K} \\ 0, & K \neq \bar{K} .\end{cases}
$$

Proof. We have $x=r \cdot \exp (i \theta)$, where $r$ and $\theta$ are amplitude and phase of $x$. Since $x$ is a standard normal random variable, $r$ and $\theta$ are independent with Rayleigh and Uniform distributions, respectively [9]. Therefore, we have

$$
\begin{aligned}
& E\left\{|x|^{2 L}(x)^{K}\left(x^{*}\right)^{\bar{K}}\right\} \\
& \quad=E_{r}\left\{r^{2 L+K+\bar{K}}\right\} E_{\theta}\{\exp (i \theta(K-\bar{K}))\} .
\end{aligned}
$$

The second term in the right-hand side of (3) is zero when $K \neq \bar{K}$. Otherwise if $K=\bar{K}$, we have

$$
E\left\{|x|^{2 L}(x)^{K}\left(x^{*}\right)^{\bar{K}}\right\}=E_{r}\left\{r^{2(K+L)}\right\}=(K+L) !
$$

This is because the last expectation is simply the $(K+L)$ th moment of an exponential distribution.

Lemma 2. For any nonnegative integer numbers $k_{i, j}$ 's and $K_{i}$ 's, one has

$$
\sum_{\substack{k_{i, 1}+k_{i, 2}+\cdots+k_{i, N}=K_{i} \\
K_{i}=K_{i}, \text { for } i=1, \ldots, N}} \prod_{i=1}^{N}\left(\begin{array}{c}
K_{i} \\
k_{i, 1}, \ldots, k_{j, N}
\end{array}\right)=\left(\begin{array}{c}
K \\
K_{1}, \ldots, K_{N}
\end{array}\right),
$$

where $\sum_{i=1}^{N} K_{i}=K, K_{i}=\sum_{j=1}^{N} k_{i, j}, \bar{K}_{i}=\sum_{j=1}^{N} k_{j, i}$, and $\left({ }_{K_{1}, \ldots, K_{N}}^{K}\right)=K ! / K_{1} ! \cdots K_{N} !$ is the multinomial coefficient.

Proof. The lemma is a generalization to Vandermonde's identity [10]. For a positive integer number $N$, nonnegative integers $K_{i}, k_{i, 1}, \ldots, k_{i, N}$, and real numbers $x_{i} \in \mathbb{R}(i=$ $1, \ldots, N)$, it holds that

$$
\begin{array}{r}
\left(\sum_{j=1}^{N} x_{j}\right)^{K_{i}}=\sum_{k_{i, 1}+\cdots+k_{i, N}=K_{i}}\left(\begin{array}{c}
K_{i} \\
k_{i, 1}, \ldots, k_{i, N}
\end{array}\right) \prod_{j=1}^{N} x_{j}^{k_{i, j}}, \\
i=1, \ldots, N .
\end{array}
$$

We can rewrite (10) as

$$
\begin{aligned}
& \left(\sum_{j=1}^{N} x_{j}\right)^{K}=\prod_{i=1}^{N}\left(\sum_{j=1}^{N} x_{j}\right)^{K_{i}} \\
& =\sum_{\substack{k_{i, 1}+\cdots+k_{i, N}=K_{i} \\
i=1, \ldots, N}}\left[\prod_{i=1}^{N}\left(\begin{array}{c}
K_{i} \\
k_{i, 1}, \ldots, k_{i, N}
\end{array}\right)\right] \prod_{j=1}^{N} x_{j}^{\bar{K}_{j}} .
\end{aligned}
$$

We can then directly expand the left-hand side of (11) as

$$
\left(\sum_{j=1}^{N} x_{j}\right)^{K}=\sum_{K_{1}, K_{2}, \ldots, K_{N}=K}\left(\begin{array}{c}
K \\
K_{1}, \ldots, K_{N}
\end{array}\right) \prod_{j=1}^{N} x_{j}^{K_{j}}
$$

By finding the coefficient of $\prod_{i=1}^{N} x_{j}^{K_{i}}$ in (11) and (12), and setting them equal to each other, the lemma is proved.

Lemma 3. For any set of nonnegative integer numbers $L_{i}$ 's, $K_{i}$ 's $(i=1, \ldots, N)$, and $L$, and any integer number $N \geq 2$, the following equation holds:

$$
\sum_{L_{1}+L_{2}+\cdots+L_{N}=L} \prod_{i=1}^{N}\left(\begin{array}{c}
K_{i}+L_{i} \\
L_{i}
\end{array}\right)=\left(\begin{array}{c}
N+L+K-1 \\
L
\end{array}\right),
$$

where $\sum_{i=1}^{N} K_{i}=K$. 
Proof. This is also a generalization to Vandermonde's identity. Given a positive integer number $N$, nonnegative integers $K_{i}$, $L_{i}(i=1, \ldots, N)$, and a real number $x \in \mathbb{R}$, we have

$$
\frac{1}{(1-x)^{K_{i}+1}}=\sum_{L_{i}=0}^{\infty}\left(\begin{array}{c}
K_{i}+L_{i} \\
L_{i}
\end{array}\right) x^{L_{i}}, \quad i=1, \ldots, N .
$$

Also, (14) is the geometric series formula. We can write the products of (14) for all values of $i$ as

$$
\begin{aligned}
\frac{1}{(1-x)^{K+N}} & =\prod_{i=1}^{N} \frac{1}{(1-x)^{K_{i}+1}} \\
& =\sum_{L_{1}, \ldots, L_{N}=0}^{\infty}\left(\prod_{i=1}^{N}\left(\begin{array}{c}
K_{i}+L_{i} \\
L_{i}
\end{array}\right)\right) x^{L_{1}+\cdots+L_{N}} .
\end{aligned}
$$

The left-hand side of (15) can be directly expanded as

$$
\frac{1}{(1-x)^{K+N}}=\sum_{L=0}^{\infty}\left(\begin{array}{c}
N+L+K-1 \\
L
\end{array}\right) x^{L} .
$$

By obtaining $\prod_{i=1}^{N} x_{j}^{K_{i}}$ in (15) and (16), the lemma is proved.

We are now ready for the main result of this paper as stated in the following theorem.

Theorem 4. Moments of squared norm of vector rejection of two standard complex normal vectors $\mathbf{a}, \mathbf{b} \in \mathbb{C}^{N}$ with independent entries described in (5) can be expressed as

$$
E\left\{w_{R}^{M}(\mathbf{a}, \mathbf{b})\right\}=\frac{(M+N-2) !}{(N-2) !} .
$$

Proof. We can expand $E\left\{w_{R}^{M}\right\}$ as

$$
E\left\{w_{R}^{M}(\mathbf{a}, \mathbf{b})\right\}=\sum_{m=0}^{M}\left(\begin{array}{c}
M \\
m
\end{array}\right)(-1)^{M-m} \varphi_{\mathbf{a}, \mathbf{b}}(m, M-m),
$$

where

$$
\varphi_{\mathbf{a}, \mathbf{b}}(L, K)=E\left\{\|\mathbf{a}\|^{2 L}\left(\frac{|\langle\mathbf{a}, \mathbf{b}\rangle|}{\|\mathbf{b}\|}\right)^{2 K}\right\} .
$$

To calculate (19), we can write

$$
\begin{aligned}
\varphi_{\mathbf{a}, \mathbf{b}} & (L, K) \\
= & E\left\{\left(\sum_{m=1}^{N}\left|a_{m}\right|^{2}\right)^{L}\left(\sum_{i, j=1}^{N} b_{i}^{*} a_{i} a_{j}^{*} b_{j}\right)^{K}\|\mathbf{b}\|^{-2 K}\right\} \\
= & E_{\mathbf{b}}\left\{\mu_{\mathbf{b}}\|\mathbf{b}\|^{-2 K}\right\},
\end{aligned}
$$

where

$$
\mu_{\mathbf{b}}=E_{\mathbf{a}}\left\{\left(\sum_{n=1}^{N}\left|a_{n}\right|^{2}\right)^{L}\left(\sum_{i, j=1}^{N} b_{i}^{*} a_{i} a_{j}^{*} b_{j}\right)^{K} \mid \mathbf{b}\right\} .
$$

To simplify (21), we use the following expansions:

$$
\begin{aligned}
& \left(\sum_{n=1}^{N}\left|a_{m}\right|^{2}\right)^{L} \\
& =\sum_{L_{1}+L_{2}+\cdots+L_{N}=L}\left(\begin{array}{c}
L \\
L_{1}, L_{2}, \ldots, L_{M}
\end{array}\right)\left(\prod_{i=1}^{N}\left|a_{i}\right|^{2 L_{i}}\right), \\
& \left(\sum_{i, j=1}^{N} b_{i}^{*} a_{i} a_{j}^{*} b_{j}\right)^{K} \\
& =\sum_{k_{1,1}+\cdots+k_{N, N}=K}\left(\begin{array}{c}
K \\
k_{1,1}, k_{1,2}, \ldots, k_{N, N}
\end{array}\right) \prod_{i, j=1}^{N}\left(b_{i}^{*} a_{i} a_{j}^{*} b_{j}\right)^{k_{i, j}} \\
& =\sum_{k_{1,1}+\ldots+k_{N, N}=K}\left(\begin{array}{c}
K \\
k_{1,1}, k_{1,2}, \ldots, k_{N, N}
\end{array}\right) \prod_{i=1}^{N}\left(a_{i}\right)^{K_{i}}\left(a_{i}^{*}\right)^{\bar{K}_{i}} \\
& \cdot\left(b_{i}^{*}\right)^{K_{i}}\left(b_{i}\right)^{\overline{K_{i}}} \text {, }
\end{aligned}
$$

where $K_{i}=\sum_{j=1}^{N} k_{i, j}$ and $\bar{K}_{i}=\sum_{j=1}^{N} k_{j, i}$. Multiplying (22) and taking the expectation, based on Lemma 1 we have

$$
\begin{gathered}
\mu_{\mathbf{b}}=\sum_{\substack{k_{1,1}+\cdots+k_{N, N}=K \\
K_{n}=\bar{K}_{n}, \text { for } n=1, \ldots, N}} \sum_{L_{1}+\cdots+L_{N}=L}\left(\begin{array}{c}
K \\
k_{1,1}, \ldots, k_{N, N}
\end{array}\right)\left(\begin{array}{c}
L \\
L_{1}, \ldots, L_{N}
\end{array}\right) \\
\cdot\left(\prod_{i=1}^{N}\left(K_{i}+L_{i}\right) !\left|b_{i}\right|^{2 K_{i}}\right)=L ! K ! \sum_{K_{1}+\cdots+K_{N}=K}\left\{\left(\prod_{i=1}^{N}\left|b_{i}\right|^{2 K_{i}}\right)\right. \\
\cdot \sum_{\substack{L_{1}+\cdots+L_{N}=L i=1 \\
\prod_{i}}}^{N}\left(\begin{array}{c}
K_{i}+L_{i} \\
L_{i}
\end{array}\right) \\
\left.\sum_{\substack{k_{n, 1}+k_{n, 2}+\cdots+k_{n, N}=K_{n} \\
K_{n}=\bar{K}_{n}, \text { for } n=1, \ldots, N}}^{N} \prod_{i=1}^{N}\left(\begin{array}{c}
K_{i} \\
k_{i, 1}, \ldots, k_{i, N}
\end{array}\right)\right\} .
\end{gathered}
$$

Based on Lemmas 2 and 3, (23) can be further simplified as

$$
\begin{aligned}
\mu_{\mathbf{b}} & =K ! \frac{(N+L+K-1) !}{(N+K-1) !} \\
& \cdot \sum_{K_{1}+\cdots+K_{N}=K}\left(\begin{array}{c}
K \\
K_{1}, \ldots, K_{N}
\end{array}\right)\left(\prod_{i=1}^{N}\left|b_{i}\right|^{2 K_{i}}\right) \\
& =K ! \frac{(N+L+K-1) !}{(N+K-1) !}\|\mathbf{b}\|^{2 K} .
\end{aligned}
$$

Substituting (24) into (20) gives

$$
\begin{aligned}
\varphi_{\mathbf{a}, \mathbf{b}}(L, K) & =E_{\mathbf{b}}\left\{K ! \frac{(N+L+K-1) !}{(N+K-1) !}\|\mathbf{b}\|^{2 K}\|\mathbf{b}\|^{-2 K}\right\} \\
& =K ! \frac{(N+L+K-1) !}{(N+K-1) !} .
\end{aligned}
$$


As a result, the $M$ th moment of the squared norm of the vector rejection in (18) can be obtained as

$$
\begin{aligned}
E\left\{w_{R}^{M}(\mathbf{a}, \mathbf{b})\right\} & =\sum_{m=0}^{M}\left(\begin{array}{c}
M \\
m
\end{array}\right)(-1)^{M-m} \varphi_{\mathbf{a}, \mathbf{b}}(m, M-m) \\
& =M ! \sum_{m=0}^{M}(-1)^{M-m}\left(\begin{array}{c}
N+M-1 \\
m
\end{array}\right) .
\end{aligned}
$$

Based on alternative sum of the binomial coefficients which can simply be proved by induction, we have

$$
E\left\{w_{R}^{M}(\mathbf{a}, \mathbf{b})\right\}=\frac{(M+N-2) !}{(N-2) !} .
$$

Corollary 5. The norm of the vector rejection of two standard complex normal vectors $\mathbf{a}, \mathbf{b} \in \mathbb{C}^{N}$ with independent entries described in (5) is a Nakagami random variable; that is,

$$
\left\|\operatorname{rej}_{\mathbf{b}} \mathbf{a}\right\| \sim \operatorname{Nakagami}(N-1, N-1) .
$$

Proof. Based on Theorem 4, the moment generating function (MGF) of $w_{R}(\mathbf{a}, \mathbf{b})$ can be obtained as

$$
\begin{aligned}
\mathscr{M}_{w_{R}}(t) & =E\left\{\exp \left(t w_{R}\right)\right\}=\sum_{m=0}^{\infty} E\left\{w_{R}^{m}\right\} \frac{(t)^{m}}{m !} \\
& =\sum_{m=0}^{\infty} \frac{(m+N-2) !}{(N-2) !} \frac{(t)^{m}}{m !} \\
& =\sum_{m=0}^{\infty}(N-1)_{m} \frac{(t)^{m}}{m !}=(1-t)^{1-N}, \quad t<1 .
\end{aligned}
$$

The series in (29) is the well-known Negative Binomial Series [11] and its convergence can be easily proven with the ratio test. This is the same as the MGF of the Gamma distribution with scale parameter of " 1 " and shape parameter of " $N-1$ ". Since the MGF fully describes a random distribution, we have

$$
w_{R}(\mathbf{a}, \mathbf{b}) \sim \operatorname{Gamma}(N-1,1) .
$$

Since $\left\|\mathrm{rej}_{\mathbf{b}} \mathbf{a}\right\|=\sqrt{w_{R}(\mathbf{a}, \mathbf{b})}$, the corollary is proved.

Corollary 6. The norm of the vector projection of two standard complex normal vectors $\mathbf{a}, \mathbf{b} \in \mathbb{C}^{N}$ with independent entries described in (1) is a Rayleigh random variable with parameter of “ 0.5 ".

Proof. Based on (19) in Theorem 4, the moments of $w_{P}(\mathbf{a}, \mathbf{b})$ can be obtained as

$$
E\left\{w_{R}^{M}(\mathbf{a}, \mathbf{b})\right\}=\varphi_{\mathbf{a}, \mathbf{b}}(0, M)=M ! .
$$

Therefore, the MGF of $w_{P}(\mathbf{a}, \mathbf{b})$ can be expressed as

$$
\begin{aligned}
\mathscr{M}_{w_{P}}(t) & =E\left\{\exp \left(t w_{P}\right)\right\}=\sum_{m=0}^{\infty} E\left\{w_{P}^{m}\right\} \frac{(t)^{m}}{m !} \\
& =(1-t)^{-1}, \quad t<1 .
\end{aligned}
$$

This is the same as the MGF of an exponential distribution with the parameter of " 1 ". That is,

$$
w_{P}(\mathbf{a}, \mathbf{b}) \sim \exp (1)
$$

and since $\left\|\operatorname{proj}_{\mathbf{b}} \mathbf{a}\right\|=\sqrt{w_{P}(\mathbf{a}, \mathbf{b})}$, the corollary is proved.

\section{Conflict of Interests}

The authors declare that there is no conflict of interests regarding the publication of this paper.

\section{References}

[1] H. Fox and W. Bolton, Mathematics for Engineers and Technologists, Butterworth-Heinemann, 2002.

[2] P. C. Clemmow, The Plane Wave Spectrum Representation of Electromagnetic Fields, International Series of Monographs in Electromagnetic Waves, Elsevier, 2013.

[3] J. G. Proakis, Digital Communications, McGraw-Hill, New York, NY, USA, 4th edition, 2001.

[4] A. Papoulis, Probability, Random Variables and Stochastic Processes, McGraw-Hill, New York, NY, USA, 2nd edition, 1984.

[5] T. S. Rappaport, Wireless Communications: Principles and Practice, Prentice-Hall, Upper Saddle River, NJ, USA, 1996.

[6] C. Perwass, Geometric Algebra with Applications in Engineering, Springer, Heidelberg, Germany, 2009.

[7] C. Doran and A. Lasenby, Eds., Geometric Algebra for Physicists, Cambridge University Press, 2003.

[8] J. C. Harsanyi and C.-I. Chang, "Hyperspectral image classification and dimensionality reduction: an orthogonal subspace projection approach," IEEE Transactions on Geoscience and Remote Sensing, vol. 32, no. 4, pp. 779-785, 1994.

[9] P. Beckmann, "Statistical distribution of the amplitude and phase of a multiply scattered field," Journal of Research of the National Bureau of Standards D, vol. 66, no. 3, pp. 231-240, 1962.

[10] T. Koshy, Catalan Numbers with Applications, Oxford University Press, 2009.

[11] E. W. Weisstein, "Negative binomial series," Wolfram MathWorld. 


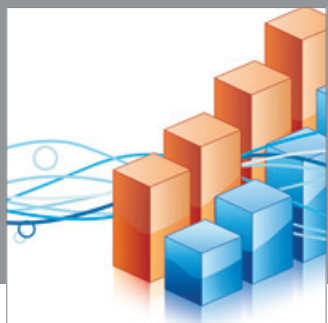

Advances in

Operations Research

mansans

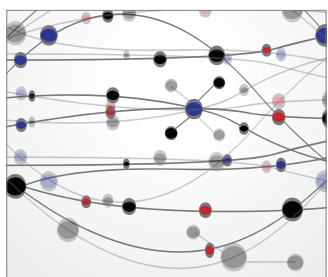

The Scientific World Journal
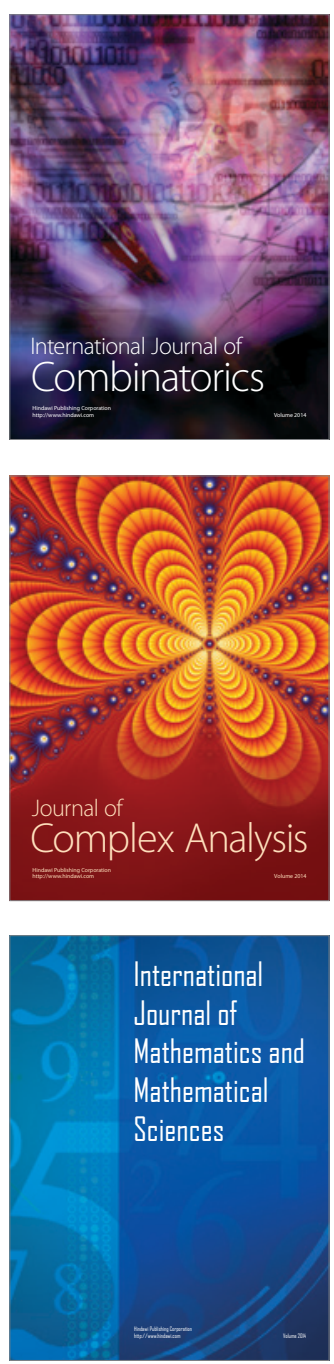
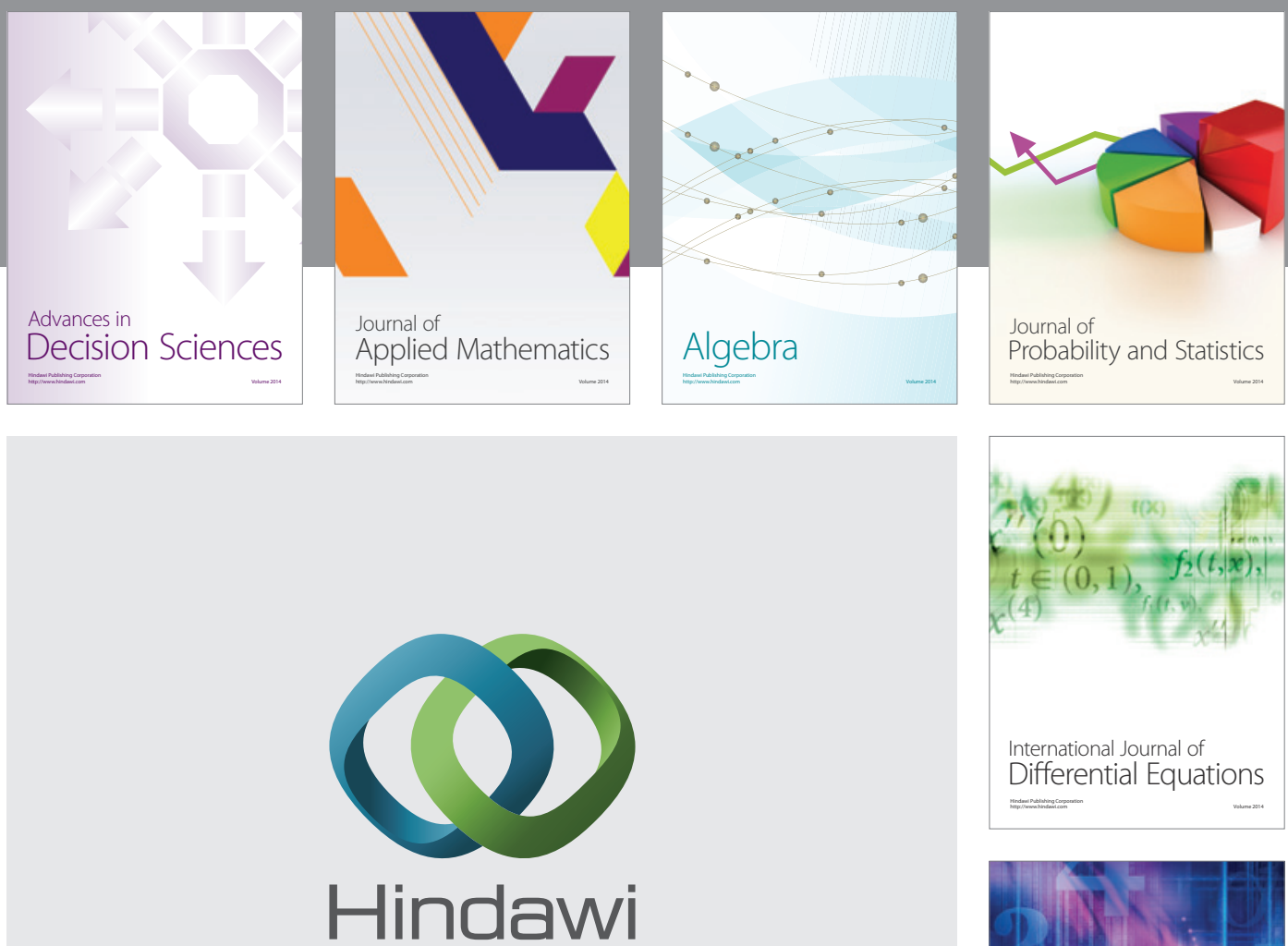

Submit your manuscripts at http://www.hindawi.com
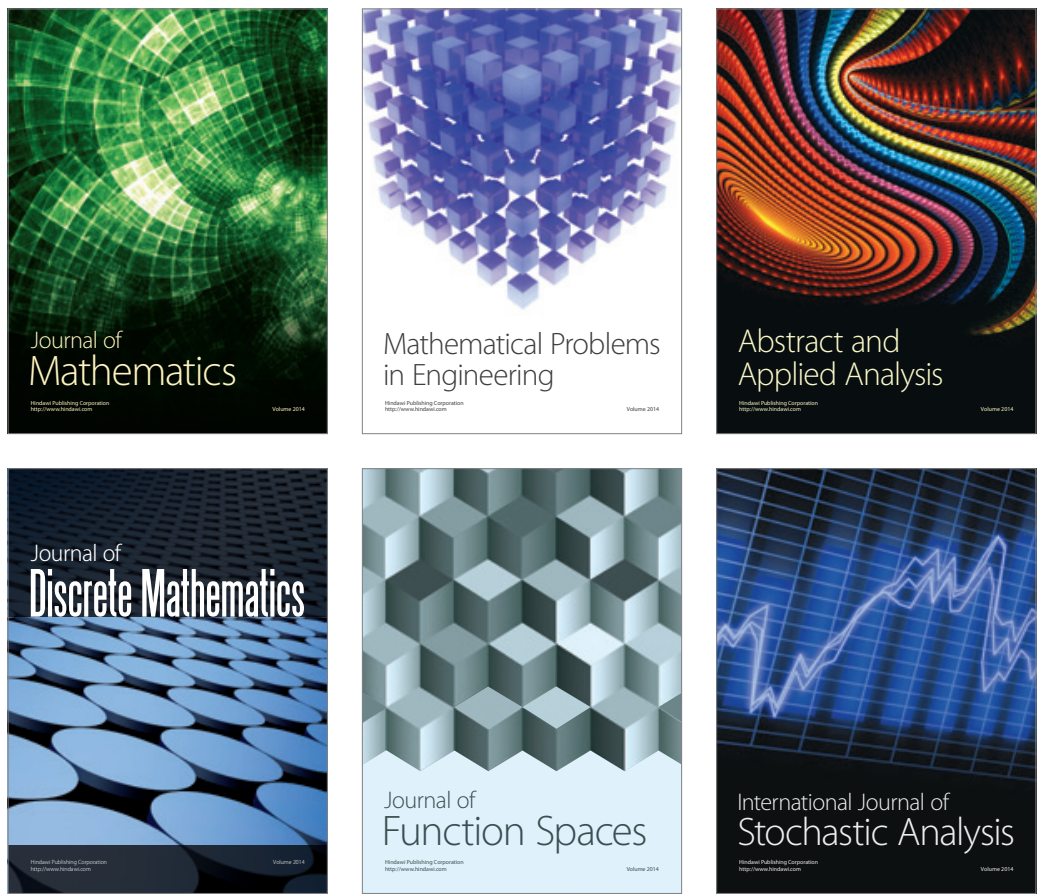

Journal of

Function Spaces

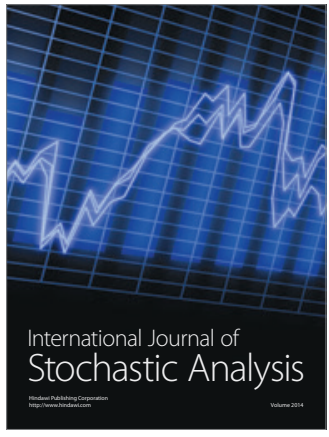

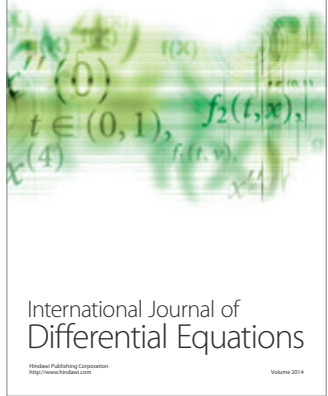
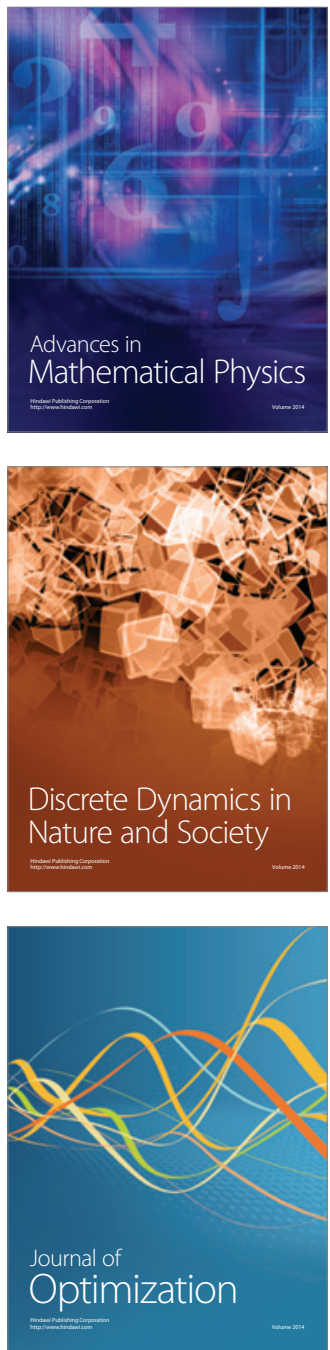